\title{
Prediction of three-month readmission based on haematological parameters in patients with severe COPD exacerbation
}

\begin{abstract}
Introduction: Approximately one-third of patients hospitalised for an exacerbation of chronic obstructive pulmonary disease (COPD) are readmitted to the hospital within 90 days. It is of interest to identify biomarkers that predict relapse in order to prevent readmission in these patients. In our prospective study of patients admitted for COPD exacerbation, we aimed to analyse whether routine haematological parameters can help predict the three-month readmission risk.

Material and methods: 106 patients were included, of whom 23 were female (22\%). The age (mean \pm SD) was $73 \pm 10$ years, and the forced expiratory volume in 1 second $\left(\mathrm{FEV}_{1}\right)$ was $44 \pm 15 \%$. The haematological parameters were obtained from the first blood test result during admission. The variables were as follows: red cell distribution width, mean platelet volume (MPV), platelet (PLT) count, neutrophil to lymphocyte ratio, PLT to lymphocyte ratio, MPV to PLT ratio, and eosinophil count. Patients were differentiated into two groups for each haematological parameter according to median value, and the percentage of readmissions in each of the groups was recorded.

Results: Twenty-five patients (24\%) were readmitted to hospital within three months of discharge. Only the difference in low-MPV and high-MPV patients was significant ( $37 \%$ vs $10 \%, \mathrm{p}=0.001)$. The predictive capacity for three-month readmission measured by the area under the curve (AUC) did not show clinically applicable values; the best result was for MPV (AUC 0.64). In the remaining values, the AUC was between 0.52 and 0.55 .

Conclusion: Routine haematological parameters proposed as prognostic biomarkers in COPD obtained at the moment of hospital admission were not useful for predicting three-month readmission.
\end{abstract}

Key words: biomarkers, COPD, prognosis, readmission

Adv Respir Med. 2021; 89: 501-504

\section{Introduction}

Severe exacerbations of chronic obstructive pulmonary disease (COPD), defined as those leading to hospitalization, are associated with high mortality, a negative impact on the quality of life, and a significant burden on the healthcare system [1]. Importantly, between $12-45 \%$ of patients hospitalised for exacerbations of COPD are readmitted to the hospital within 90 days [2, 3]. If patients at an increased risk can be detected, a personally tailored post-discharge plan may potentially reduce readmissions [4]. Consequently, it is of interest to identify biomarkers in order to prevent readmission of these patients [2]. However, although COPD exacerbation prediction tools have been proposed [5], no clear consensus exists on markers that can predict readmission [3].

A small number of studies, mainly retrospective, have evaluated the role of routine haematological parameters in different circumstances as prognostic biomarkers in COPD patients (including the prediction of the risk of readmission) with sometimes contradictory results. These easily accessible and inexpensive tests include: red blood cell distribution width (RDW), mean platelet volume (MPV), platelet (PLT) count, neutrophil to lymphocyte ratio, the PLT to lymphocyte ratio, the MPV to PLT ratio, and the number of eosinophils [6-11].

In a prospective study of patients admitted for COPD exacerbations, we aimed to analyse

Address for correspondence: Eduardo Garcia-Pachon, Department of Respiratory Medicine, Hospital General Universitario de Elche, Elche, Alicante, Spain; e-mail: egpachon@gmail.com DOI: 10.5603/ARM.a2021.0076 | Received: 04.01.2021 | Copyright (C) 2021 PTChP | ISSN 2451-4934 | e-ISSN 2543-6031

This article is available in open access under Creative Common Attribution-Non-Commercial-No Derivatives 4.0 International (CC BY-NC-ND 4.0) license, allowing to download articles and share them with others as long as they credit the authors and the publisher, but without permission to change them in any way or use them commercially. 
Table 1. Percentage of three-month readmission with haematological parameters

\begin{tabular}{lcccc}
\hline Parameter & Median value & Low & High & P-value \\
\hline Red cell distribution width [\%] & 15.5 & $18 \%$ & $31 \%$ & 0.103 \\
Mean platelet volume [fL] & 9.0 & $37 \%$ & $10 \%$ & 0.001 \\
Platelets [× $\left.10^{9} / \mathrm{L}\right]$ & 226 & $25 \%$ & $23 \%$ & 0.95 \\
Neutrophil to lymphocyte ratio & 8.56 & $28 \%$ & $21 \%$ & 0.87 \\
Platelet to lymphocyte ratio & 0.21 & $26 \%$ & $22 \%$ & 0.97 \\
MPV to platelet ratio & 38.4 & $24 \%$ & $23 \%$ & 0.99 \\
Eosinophils [cells $/ \mu \mathrm{L}$ ] & 120 & $25 \%$ & & 0.95 \\
\hline
\end{tabular}

Low: Below or equal to median value. High: Above the median value

whether routine haematological parameters obtained at the time of admission can help to predict three-month readmission.

\section{Material and methods}

The study was approved by the local ethics committee and the patients signed an informed consent form (reference PI 31/2019). Our study initially included 115 consecutive patients admitted because of exacerbation of COPD in a respiratory ward of a university hospital over a 14-month period. Only the first episode was taken into consideration if more than one occurred for the same patient. Nine patients were excluded; six because of death, two because no follow-up was available, and one due to leukaemia. 106 patients completed the three-month follow-up since discharge and were included in the study. Of these participants, 23 were female (22\%) and 83 were male $(78 \%)$. The average age (mean \pm SD) was $73 \pm 10$ years, and the forced expiratory volume in 1 second $\left(\mathrm{FEV}_{1}\right)$ was $44 \pm 15 \%$ of predicted value. Of those patients, $63(59 \%)$ had at least one admission in the previous year. The haematological parameters were obtained from the first blood test during admission, as per clinical practice. Blood analysis was performed in the centralized laboratory with the analyser model Vitros 5600 (Ortho Clinical Diagnostics, Raritan, NJ, USA). The variables included for evaluation were as follows: RDW, MPV, platelet (PLT) count, neutrophil to lymphocyte ratio, PLT to lymphocyte ratio, MPV to PLT ratio, and eosinophil count. Patients were differentiated into two groups for each haematological parameter: those with low values (below or equal to the median) and those with high values (above the median of the parameter). The percentage of three-month readmissions in each of the groups was recorded.
A chi-squared test was performed to compare categorical variables, and a Mann-Whitney U test was used to compare quantitative variables. The discriminating performance of the parameters was evaluated using receiver operating characteristic (ROC) curves with corresponding areas under the curve (AUC). Statistical analysis was performed using the programs SPSS version 22 (IBM Corp., Armonk, NY, EEUU) and the R statistics package (www.r-project.org). A p-value below 0.05 was considered statistically significant.

\section{Results}

Twenty-five of the 106 patients (24\%) were readmitted to hospital within three months of discharge for a new COPD exacerbation. Readmissions occurred in a median of 41 days (interquartile range [IR], 20-68 days). Of these 25 patients, $22(88 \%)$ had at least one admission in the previous year. Of the remaining (non-readmitted) 81 patients, 41 (51\%) had been admitted in the previous year $(\mathrm{p}<0.001)$. Compared with those not requiring readmission, patients with readmission had a higher number of admissions in the previous year (median, IR: 3.0 [1.0-4.3] vs $0.0[0.0-2.0, \mathrm{p}<0.001]$ and lower $\mathrm{FEV}_{1}$ in terms of percentage of predicted value (36\% [29-48] vs $45 \%$ [34-55], $p=0.048$ ). There were no significant differences in age, gender, or duration of admission.

The percentage of patients who were readmitted in the first three months after discharge was calculated for each haematological parameter. As shown in Table 1, only the difference in low-MPV and high-MPV patients was significant. The predictive capacity for three-month readmission of each parameter measured by the AUC did not show clinically applicable values; the best result was for MPV (AUC 0.64, Figure 1). 


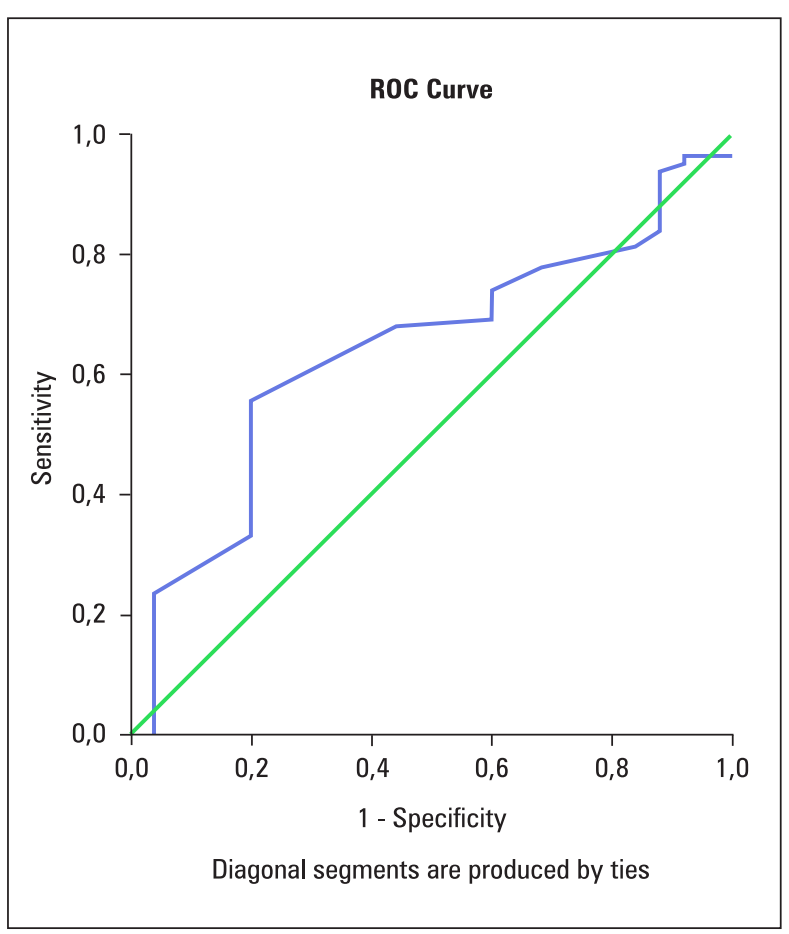

Figure 1. Area under the curve for predicting three-month readmission with mean platelet volume

In the remaining values, the AUC was between 0.52 and 0.55 .

\section{Discussion}

Numerous parameters of routine blood tests are considered to be underlying biomarkers of inflammation and potentially useful in establishing a prognosis in patients with COPD [12]. These tests have the advantage of being inexpensive and immediately available for clinicians. Therefore, the potential contribution of their possible clinical application is of great interest. In retrospective studies, RDW was an independent risk factor for readmission [6]. MPV, although less frequently evaluated, has shown inconclusive results [7]. The neutrophil to lymphocyte ratio and the PLT to lymphocyte ratio are both elevated in stable COPD patients, and their values further increased during exacerbations $[9,10]$. These ratios have been identified as being able to predict COPD progression and outcomes such as hospital mortality [9, 10]. Eosinophil counts have been proposed as being predictors of the one-year risk of hospitalisation [11]. Consequently, we considered it necessary to study the possibility that these parameters would offer prognostic information in patients admitted for acute COPD. Theoretically, the values of these indices obtained in a blood test at the time of ad- mission could indicate the risk of readmission in the three months after discharge and thus, enable specific therapeutic plans.

Unfortunately, in our prospective study of consecutive COPD patients, we have not been able to confirm any of the promising results of these parameters obtained at the moment of hospital admission. Only MPV showed significant differences between patients with and without readmission. However, in view of the AUC results, its values do not allow for its application in individual cases. MPV is a component of routine blood analysis that correlates highly with platelet activation and is linked to the pathophysiology of the inflammatory process during COPD exacerbations [7]. In fact, previous studies have shown that MPV may represent an inflammatory marker for multiple conditions such as vascular diseases, rheumatoid diseases, and diabetes mellitus [7]. Platelet distribution width (PDW) is an additional parameter with prognostic value, but was not available in our study [13]. In our series, as described in other studies [2], hospitalisation in the year prior to index admission was the key predictor of COPD-related readmission. Although conducted prospectively and applied in a real clinical setting, our work has the limitation of having been conducted in a single centre, so the results should be confirmed in other series. An additional limitation of this study is the absence of a definition of the probable causes of exacerbation (infectious vs non-infectious). This data could have allowed an analysis according to the cause of admission and would perhaps have detected subgroups in which biomarker analysis could be more useful. New research continues to be undertaken on biomarkers and factors that can help clinicians predict subsequent exacerbations of COPD.

\section{Conclusions}

The routine haematological parameters proposed as prognostic biomarkers in COPD, when obtained at the moment of hospital admission, were not useful for predicting three-month readmission.

\section{Conflict of interest}

Authors have no conflict of interest to declare.

\section{References:}

1. Halpin DMg, Miravitlles M, Metzdorf N, et al. Impact and prevention of severe exacerbations of COPD: a review of the evidence. Int J Chron Obstruct Pulmon Dis. 2017; 12: 2891-2908, doi: 10.2147/COPD.S139470, indexed in Pubmed: 29062228. 
2. Njoku CM, Alqahtani JS, Wimmer BC, et al. Risk factors and associated outcomes of hospital readmission in COPD: A systematic review. Respir Med. 2020; 173: 105988, doi: 10.1016/j. rmed.2020.105988, indexed in Pubmed: 33190738.

3. Kong CW, Wilkinson TMA. Predicting and preventing hospital readmission for exacerbations of COPD. ERJ Open Res. 2020 6(2): 00325-2019, doi: 10.1183/23120541.00325-2019, indexed in Pubmed: 32420313.

4. Hegelund A, Andersen IC, Andersen MN, et al. The impact of a personalised action plan delivered at discharge to patients with COPD on readmissions: a pilot study. Scand J Caring Sci. 2020; 34(4): 909-918, doi: 10.1111/scs.12798, indexed in Pubmed: 31865631.

5. Adibi A, Sin D, Safari A, et al. The acute COPD exacerbation prediction tool (ACCEPT): a modelling study. Lancet Respir Med. 2020; 8(10): 1013-1021, doi: 10.1016/s22132600(19)30397-2, indexed in Pubmed: 32178776.

6. Epstein D, Nasser R, Mashiach T, et al. Increased red cell distribution width: A novel predictor of adverse outcome in patients hospitalized due to acute exacerbation of chronic obstructive pulmonary disease. Respir Med. 2018; 136: 1-7, doi: 10.1016/i. rmed.2018.01.011, indexed in Pubmed: 29501240.

7. Ma Y, Zong D, Zhan Z, et al. Feasibility of mean platelet volume as a biomarker for chronic obstructive pulmonary disease A systematic review and meta-analysis. J Int Med Res. 2019; 47(12): 5937-5949, doi: 10.1177/0300060519887886, indexed in Pubmed: 31774003.
8. Mallah H, Ball S, Sekhon J, et al. Platelets in chronic obstructive pulmonary disease: An update on pathophysiology and implications for antiplatelet therapy. Respir Med. 2020; 171 106098, doi: 10.1016/j.rmed.2020.106098, indexed in Pubmed: 32777683

9. Paliogiannis P, Fois AG, Sotgia S, et al. Neutrophil to lymphocyte ratio and clinical outcomes in COPD: recent evidence and future perspectives. Eur Respir Rev. 2018; 27(147): 170113 , doi: 10.1183/16000617.0113-2017, indexed in Pubmed: 29436405 .

10. El-Gazzar AG, Kamel MH, Elbahnasy OK, et al. Prognostic value of platelet and neutrophil to lymphocyte ratio in COPD patients. Expert Rev Respir Med. 2020; 14(1): 111-116, doi: 10.1080/17476348.2019.1675517, indexed in Pubmed: 31577911.

11. Couillard S, Larivée P, Courteau J, et al. Eosinophils in COPD exacerbations are associated with increased readmissions. Chest. 2017; 151(2): 366-373, doi: 10.1016/j.chest.2016.10.003, indexed in Pubmed: 27746201.

12. Xiong W, Xu M, Zhao Y, et al. Can we predict the prognosis of COPD with a routine blood test? Int J Chron Obstruct Pulmon Dis. 2017; 12: 615-625, doi: 10.2147/COPD.S124041, indexed in Pubmed: 28243079.

13. Białas AJ, Pedone C, Piotrowski WJ, et al. Platelet distribution width as a prognostic factor in patients with COPD - pilot study. Int J Chron Obstruct Pulmon Dis. 2017; 12: 2261-2267, doi: 10.2147/COPD.S131868, indexed in Pubmed: 28814854. 